\title{
Prognostic Factors in Patients with Non-small Cell Lung Carcinoma and Brain Metastases: a Malaysian Perspective
}

\author{
Weng Heng Tang ${ }^{1 *}$, Adlinda Alip ${ }^{1}$, Marniza Saad ${ }^{1}$, Vincent Chee Ee Phua ${ }^{1}$, Hari \\ Chandran', Yi Hang Tan ${ }^{3}$, Yan Yin Tan ${ }^{3}$, Voon Fong Kua ${ }^{3}$, Mohamed Ibrahim \\ Wahid $^{3}$, Lye Mun Tho ${ }^{3}$
}

\begin{abstract}
Background: Brain metastases occur in about $20-40 \%$ of patients with non-small-cell lung carcinoma (NSCLC), and are usually associated with a poor outcome. Whole brain radiotherapy (WBRT) is widely used but increasingly, more aggressive local treatments such as surgery or stereotactic radiosurgery (SRS) or stereotactic radiotherapy (SRT) are being employed. In our study we aimed to describe the various factors affecting outcomes in NSCLC patients receiving local therapy for brain metastases. Materials and Methods: The case records of 125 patients with NSCLC and brain metastases consecutively treated with radiotherapy at two tertiary centres from January 2006 to June 2012 were analysed for patient, tumour and treatment-related prognostic factors. Patients receiving SRS/SRT were treated using Cyberknife. Variables were examined in univariate and multivariate testing. Results: Overall median survival was 3.4 months (95\% CI: 1.7-5.1). Median survival for patients with multiple metastases receiving WBRT was 1.5 months, 1-3 metastases receiving WBRT was 3.6 months and 1-3 metastases receiving surgery or SRS/SRT was 8.9 months. ECOG score $(\leq 2 v s>2, p=0.001)$, presence of seizure (yes versus no, $p=0.031$ ), treatment modality according to number of brain metastases (1-3 metastases+surgery or SRS/SRT \pm WBRT $v s$ 1-3 metastases+WBRT only $v s$ multiple metastases+WBRT only, $p=0.007$ ) and the use of post-therapy systemic treatment (yes versus no, $\mathrm{p}=0.001$ ) emerged as significant on univariate analysis. All four factors remained statistically significant on multivariate analysis. Conclusions: ECOG $\leq 2$, presence of seizures, oligometastatic disease treated with aggressive local therapy (surgery or SRS/SRT) and the use of post-therapy systemic treatment are favourable prognostic factors in NSCLC patients with brain metastases.
\end{abstract}

Keywords: Non-small cell lung carcinoma - brain metastases - stereotactic radiosurgery - Cyberknife

Asian Pac J Cancer Prev, 16 (5), 1901-1906

\section{Introduction}

Patients with non-small cell lung carcinoma (NSCLC) develop brain metastases (BM) in about $20-40 \%$ of cases (Law et al., 2001; Ceresoli et al., 2002). With modern imaging techniques for detecting $\mathrm{BM}$ and prolonged survival of lung cancer patients due to improvements in both local and systemic approaches, the prevalence of BM is set to increase (Langley et al., 2013). Patients with BM from an underlying lung primary seem to fare less well in terms of overall survival compared with other primary tumour sites, such as breast and colonic malignancies, with a median survival of less than 3 months (Priestman et al., 1996; Sperduto et al., 2010; Natukula et al., 2013). This poses a difficult management conundrum.

The management of these patients may include surgical resection, radiation therapy, or systemic therapy. The largest randomized trial to date looking at the role of WBRT in NSCLC showed no overall survival benefit and no improvement in quality of life (Langley et al., 2013). At the same time, WBRT can negatively impact quality of life (Chang et al., 2009). Surgical resection of brain metastases may reduce death from neurological causes and may offer improved functional independent survival (Hart et al., 2005). An alternative to open surgical resection is stereotactic radiosurgery (if delivered in single session) or stereotactic radiotherapy (usually between 2 to 5 sessions) where very high doses of radiation are delivered precisely to the tumour in an attempt to ablate the lesions. There is accumulating evidence to suggest that SRS/SRT offers improved local control and quality of life and in some clinical situations better survival (Patil et al., 2012). However these aggressive local therapies are not without risks, therefore patient selection is vital to ensure suitable patients are considered for the treatment to achieve potential improved outcome.

Finally to ascertain the benefits of the various treatment approaches for brain metastases, studies should aim to

${ }^{1}$ Clinical Oncology Unit, ${ }^{2}$ Neurosurgical Unit, University of Malaya Medical Centre, Kuala Lumpur, ${ }^{3}$ Beacon Cancer Hospital, Petaling Jaya, Malaysia *For correspondence: tangwengheng@ummc.edu.my 
Weng Heng Tang et al

control for various patient and clinical characteristics such as comorbidites and performance status. In our study, we aim to analyse outcomes from two tertiary institutions where NSCLC patients with brain metastases received treatment, and describe the various prognostic and treatment factors affecting outcomes.

\section{Materials and Methods}

Patient records from January 2006 to June 2012 were reviewed from two large Malaysian tertiary referral cancer centres - University of Malaya Medical Centre and Beacon
Cancer Hospital. 125 patients with NSCLC histology and $\mathrm{BM}$ who received radiotherapy treatment, were identified.

$\mathrm{BM}$ were diagnosed using computed tomography (CT) or magnetic resonance imaging (MRI). All patients had histological confirmation of NSCLC pathology either via biopsy of the primary malignancy or systemic metastases (including stereotactic biopsy of the brain).

WBRT radiotherapy was delivered using 6-MV linear accelerator at the University Malaya Medical Centre. Different dose/fractionation schedules were used but the most commonly employed was $20 \mathrm{~Gy}$ in 5 fractions over 5 days. SRS (for example 20Gy in 1 fraction) or SRT (30Gy

Table 1. Baseline Characteristics

\begin{tabular}{|c|c|c|c|}
\hline & & $\mathrm{N}=125$ & $\%$ \\
\hline Age & $\begin{array}{l}<65 \\
\geq 65 \\
\text { median=59 }\end{array}$ & $\begin{array}{l}81 \\
44\end{array}$ & $\begin{array}{l}64.8 \\
35.2\end{array}$ \\
\hline Sex & $\begin{array}{l}\text { male } \\
\text { female }\end{array}$ & $\begin{array}{l}67 \\
58\end{array}$ & $\begin{array}{l}53.6 \\
46.4\end{array}$ \\
\hline ECOG & $\begin{array}{l}0 \\
1 \\
2 \\
3 \\
4\end{array}$ & $\begin{array}{r}2 \\
54 \\
28 \\
36 \\
5\end{array}$ & $\begin{array}{c}1.6 \\
43.2 \\
22.4 \\
28.8 \\
4\end{array}$ \\
\hline Number of brain metastases & $\begin{array}{l}1 \\
2 \text { to } 3 \\
>3\end{array}$ & $\begin{array}{l}29 \\
35 \\
61\end{array}$ & $\begin{array}{l}23.2 \\
28 \\
48.8\end{array}$ \\
\hline Extracranial metastasis & $\begin{array}{l}\text { Yes } \\
\text { No }\end{array}$ & $\begin{array}{l}79 \\
46\end{array}$ & $\begin{array}{l}63.2 \\
36.8\end{array}$ \\
\hline Control of primary & $\begin{array}{l}\text { Yes } \\
\text { No } \\
\text { Unknown }\end{array}$ & $\begin{array}{l}19 \\
85 \\
21\end{array}$ & $\begin{array}{l}15.2 \\
68 \\
16.8\end{array}$ \\
\hline Dose fractionation of WBRT* & $\begin{array}{l}30 \mathrm{~Gy} / 10 \# / 2 \mathrm{w} \\
20 \mathrm{~Gy} / 5 \# / 1 \mathrm{w} \\
12 \mathrm{~Gy} / 2 \# / 2 \mathrm{~d}\end{array}$ & $\begin{array}{r}3 \\
108 \\
2\end{array}$ & $\begin{array}{r}2.4 \\
86.4 \\
1.6\end{array}$ \\
\hline Surgery/SRT & $\begin{array}{l}\text { Yes } \\
\text { No }\end{array}$ & $\begin{array}{l}30 \\
95\end{array}$ & $\begin{array}{l}24 \\
76\end{array}$ \\
\hline $\begin{array}{l}\text { Oligometastases }(1-3)+\text { surgery/SRT } \pm \text { WBRT } \\
\text { Oligometastases }(1-3)+\text { WBRT only } \\
\text { Multiple metastases+WBRT only }\end{array}$ & & $\begin{array}{l}30 \\
34 \\
61\end{array}$ & $\begin{array}{l}24 \\
27.2 \\
48.8\end{array}$ \\
\hline Prior systemic treatment & $\begin{array}{l}\text { Yes } \\
\text { No }\end{array}$ & $\begin{array}{l}53 \\
72\end{array}$ & $\begin{array}{l}42.4 \\
57.6\end{array}$ \\
\hline Subsequent systemic treatment & $\begin{array}{l}\text { Yes } \\
\text { No }\end{array}$ & $\begin{array}{l}39 \\
86\end{array}$ & $\begin{array}{l}31.2 \\
68.8\end{array}$ \\
\hline RPA class & $\begin{array}{l}1 \\
2 \\
3\end{array}$ & $\begin{array}{l}10 \\
63 \\
52\end{array}$ & $\begin{array}{c}8 \\
50.4 \\
41.6\end{array}$ \\
\hline GPA index & $\begin{array}{l}0-1 \\
1.5-2.5 \\
3 \\
3.5-4 \\
\end{array}$ & $\begin{array}{r}61 \\
50 \\
7 \\
7 \\
\end{array}$ & $\begin{array}{c}48.8 \\
40 \\
5.6 \\
5.6 \\
\end{array}$ \\
\hline
\end{tabular}

*Twelve patients (9.6\%), who were treated with SRT, did not have WBRT 
Prognostic Factors in Patients with Non-small Cell Lung Carcinoma and Brain Metastases: A Malaysian Perspective

in 5 fractions) was delivered using 6MV Cyberknife ${ }^{\circledR}$ at the Beacon Cancer Hospital. Not more than 5 fractions were used to deliver SRT, treatment was delivered daily on consecutive days. Corticosteroids were administered during radiotherapy, according to clinical need.

Patient demographic, tumour and treatment-related factors were documented. These included age, sex, Eastern Cooperative Oncology Group (ECOG) performance status, presence of extracranial metastasis, presence of seizures, control of primary tumour, treatment modality, number of brain metastases and the use of prior and postradiation systemic treatment. Prior prognostic assessment tools have been published for use in patients with BM. We calculated both the Recursive Partitioning Assessment (RPA) score (Gaspar et al., 1997) and Graded Prognostic Assessment (GPA) score (Sperduto et al., 2008) in our patients.

Statistical analysis was performed using SPSSv20. Overall survival was calculated from the date of initiation of treatment for brain metastases. Survival data was updated to 1 June 2014 based on Malaysian National Registration Department (NRD) database. Data was censored at last follow-up. Survival curves were plotted using Kaplan and Meier method. Two or more curves were compared by using log rank test (Mantel-Cox). Statistical significance was defined by $\mathrm{p}<0.05$. All variables were examined in univariate tests. Four independent prognostic factors found to be significant on univariate analysis were included in multivariate analysis using Cox regression method. Patient outcomes were stratified according to RPA and GPA scores. The study received approval from respective institutions' Medical Ethics Committee.

\section{Results}

According to the NRD data, 124 of the patients had died, while the remaining one was alive. Median age was 59 , male to female ratio was $54 \%$ to $46 \%$. 1-3 BM were present in $51.2 \%$ of patients and $>3$ metastases in $48.8 \%$. Amongst patients with $1-3$ metastases $(n=64), 34$ received WBRT only, whereas 30 received local therapy either surgery or SRS/SRT. All patients who had surgery received WBRT whereas 12 patients who were treated with SRS/SRT, did not have WBRT. The most common fractionation schedule amongst those receiving WBRT $(\mathrm{n}=113)$ is $20 \mathrm{~Gy}$ in 5 fractions over 5 days $(\mathrm{n}=108)$. Table 1 shows the baseline characteristics.
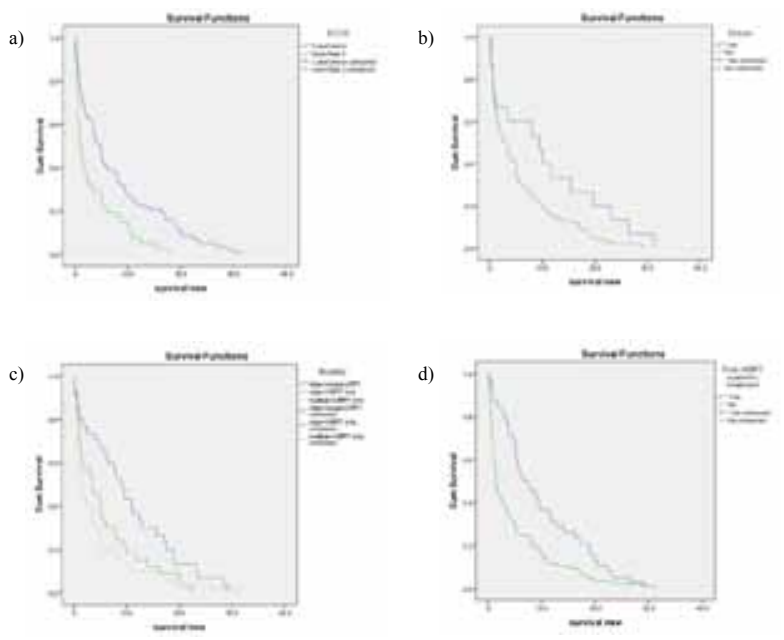

Figure 1. Kaplan-Meier survival curves according to a) ECOG score, b) presence of seizure, c) treatment modality based on number of brain metastases, and d) the use of post-therapy systemic treatment. ECOG=Eastern Cooperative Oncology Group performance status
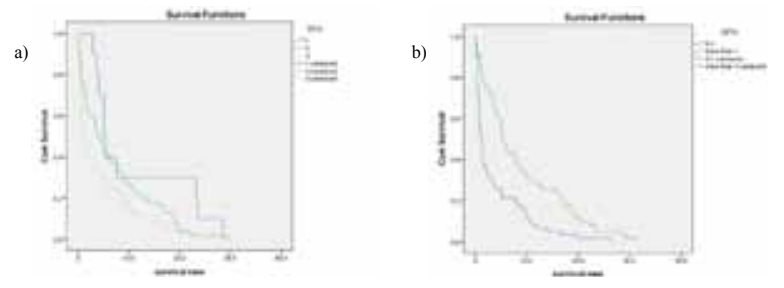

Figure 2. Kaplan-Meier Survival Curves According to a) RPA Score, and b) GPA Index

Table 2. Univariate Analysis of Overall Survival According to Prognostic Factors

\begin{tabular}{llcl}
\hline Covariates & Comparison & Median survival in months $(95 \% \mathrm{CI})$ & $\mathrm{p}$ value \\
\hline Age & $<65 v s \geq 65$ & $4.3(2.8-5.7)$ vs $1.2(0.7-1.7)$ & 0.070 \\
Sex & Male $v s$ Female & $1.9(0-4.1) v s 3.8(2.1-5.5)$ & 0.857 \\
ECOG & $\leq 2 v s>2$ & $4.8(3.4-6.2) v s 1.1(0.6-1.6)$ & $0.001^{*}$ \\
Extracranial metastasis & Yes $v s$ No & $2.3(0.6-4.0) v s 5.0(3.7-6.3)$ & 0.283 \\
Control of primary tumour & Yes $v s$ No $v s$ Unknown & $7.6(2.9-12.2) v s 2.5(1.1-3.9) v s 1.9(0-3.8)$ & 0.111 \\
Seizure & Yes $v s$ No & $9.5(1.2-17.8) v s 3.0(1.5-4.5)$ & $0.031^{*}$ \\
Treatment modality according & Oligo (1-3)+surgery/SRT \pm WBRT & $8.9(6.2-11.6) v s$ & $0.007^{*}$ \\
to number of brain metastases & Oligo (1-3)+WBRT only & $3.6(0-7.3) v s$ & \\
& Multiple+WBRT only & $1.5(0.6-2.4)$ & 0.976 \\
Prior systemic treatment & Yes $v s$ No & $3.5(2.4-4.6) v s 1.9(0-4.5)$ & $0.001^{*}$ \\
Subsequent systemic treatment & Yes $v s$ No & $7.6(3.8-11.3) v s 1.5(0.9-2.1)$ & \\
*ECOG=Eastern Cooperative Oncology Group performance status; ${ }^{*}$ p $<0.05$ & &
\end{tabular}

Table 3. Analysis of Overall Survival According to Prognostic Factors

\begin{tabular}{lccc}
\hline Covariates & Comparison & Median survival in months (95\% CI) & p value \\
\hline RPA & 1 vs 2 vs 3 & $5.2(3.7-6.8)$ vs $4.3(2.7-5.9)$ vs $1.2(0.6-1.8)$ & 0.033 \\
GPA & $\leq 1$ ss $>1$ & $1.2(0.5-1.9)$ vs 5.3 (4.3-6.3) & 0.001 \\
\hline *RPA=Recursive Partitioning Analysis score; GPA=Graded Prognostic Assessment index &
\end{tabular}


The median overall survival was 3.4 months (95\%CI: 1.7-5.1). Median survival for patients with multiple metastases receiving WBRT was 1.5 months, 1-3 metastases receiving WBRT was 3.6 months and 1-3 metastases receiving surgery or stereotactic radiosurgery/radiotherapy (SRS/SRT) was 8.9 months. Of the various prognostic factors, ECOG score ( $\leq 2 v s>2, \mathrm{p}=0.001)$, presence of seizure (yes versus no, $\mathrm{p}=0.031$ ), treatment modality according to number of brain metastases (1-3 metastases + surgery/SRT \pm WBRT $v s$ 1-3 metastases+WBRT only $v s$ multiple metastases+WBRT only, $\mathrm{p}=0.007)$ and the use of post-therapy systemic treatment (yes versus no, $\mathrm{p}=0.001$ ) emerged as significant on univariate analysis (Table 2 and Figure 1a-d). All four factors remained statistically significant on multivariate analysis using Cox regression method $(\mathrm{p}<0.05)$.

Both Recursive Partitioning Analysis (RPA) score $(\mathrm{p}=0.033)$ and Graded Prognostic Assessment (GPA) index $(\mathrm{p}=0.001)$ demonstrated survival differences according to known prognostic groupings (Table 3 and Figure 2a-b).

\section{Discussion}

To our knowledge this is the first data to emerge from the South East Asian Region (ASEAN) to describe factors involved in the survival of patients with NSCLC cancer and BM. Lung cancer is a huge burden in the ASEAN region with annual overall lung cancer incidence about 162000 (International Agency for Research on Cancer, 2014). Brain metastases incidence is estimated at 20-40\%.

In our series of NSCLC patients with brain metastases, ECOG score $\leq 2$, presence of seizure, oligometastatic disease treated with aggressive local therapy (surgery or SRS/SRT) and the use of post-therapy systemic treatment are significant independent prognostic factors for overall survival.

Performance status is a major indicator of outcomes after treatment (Gaspar et al., 1997; Sperduto et al., 2008; Cai et al., 2013). Both RPA classification, which includes three prognostic groups: I (patients < 65 years, Karnofsky Performance Status [KPS] $\geq 70$, controlled primary tumor, and no extracranial metastases), III (KPS $<70$ ), and II (all patients not in Class I or III) (Gaspar et al., 1997), and GPA index incorporate KPS as measure of outcome (Gaspar et al., 1997; Sperduto et al., 2008). In our retrospective review of patient records, ECOG score was documented where ECOG $\leq 2$ corresponding to KPS $\geq 70$ (Oken et al., 1982) has been found to be one of the strongest prognostic factors for overall survival.

Presence of seizure is a recognised favourable prognostic factor in primary brain tumours (Gehan, 1977). This may be explained with its association with low grade gliomas and not high grade gliomas. However, to our knowledge, occurrence of seizure has not been correlated with outcome in brain metastases. The improvement in median survival seen in patients with seizures requires further mechanistic explanation and might be explored in subsequent studies.

WBRT has been used to treat brain metastases for several decades. Despite this, the degree of benefit for this approach remains controversial. The only randomized trial to address the role of WBRT in NSCLC BM showed no overall survival benefit and no improvement in quality of life compared to best supportive care (Langley et al., 2013). Interestingly, the majority of the patients in that trial $(68 \%)$ presented with 3 or less metastases, which may indicate WBRT is inadequate treatment for oligometastatic disease. Furthermore WBRT is not without complications. For example, Chang et al. demonstrated a significant decline in learning and memory function by 4 months for patients receiving WBRT with SRS as opposed to SRS alone (Chang et al., 2009).

The strategy of treating BM with aggressive local therapy either surgical resection or SRS/SRT is aimed at improving local control thereby impacting overall survival. Metaanalyses show that surgical resection can reduce death from neurological causes and offer improved functional independent survival (Hart et al., 2005; Duan et al., 2014). Small randomized trial and single centre experience hint at an overall survival advantage (Patchell et al., 1990; Akhavan, 2014). Similarly SRS and SRT have been shown to improve local control and quality of life (Patil et al., 2012). Our own data suggest a significant role for local therapy in improving survival. Patients with oligometastatic disease who only received WBRT demonstrated a median survival of 3.6 months whereas those who underwent surgical resection or SRS/SRT demonstrated a median survival of 8.9 months, a 2.5 fold increase. SRS/SRT particularly, obviates the need for surgery and associated complication, and is a promising area for future research. Additionally, more studies are required to ascertain the best way to sequence or combine available therapies.

Patients with symptomatic BM are often excluded from pharmaceutical clinical trials evaluating systemic therapeutic agents. Thus, the role of such systemic treatments in treating BM is often unclear. In our study, the use of post-therapy systemic treatment effectively separates the survival curves. This observed improvement is unlikely to be due to selection bias (e.g. better ECOG performance status), as this prognostic factor remained statistically significant on multivariate analysis. The blood-brain barrier (BBB) allows only relatively low concentrations of most systemic agents to penetrate into the central nervous system (CNS). However, prior studies that have shown this have been limited to normal brain and micro-metastatic disease. In the setting of gross metastatic disease, such as in our patient population, the BBB may be disrupted by abnormal tumour vasculature and/or radiotherapy (Postmus et al., 1999; Van Vulpen et al., 2002; Liu et al., 2012; Cai et al., 2013), thus allowing greater degree of penetration of systemic agents. Furthermore, some small molecule tyrosine kinase inhibitor might work synergistically with radiotherapy leading to enhanced effectiveness (Zeng et al., 2011). Our result certainly suggests that patient who received systemic agents post BM therapy fared better. However, another explanation might be that more effective therapy such as surgery or SRS/SRT compared to WBRT improved survival thereby 
allowing the opportunity to deliver subsequent systemic therapy. Our results are an interesting clinical observation, which would be useful to validate in future studies.

In our study, both RPA and GPA scoring systems were able to identify subgroup of patients with poor outcomes. These are indices derived from studies across several thousand patients (Gaspar et al., 1997; Sperduto et al., 2008). Therefore, this serves as a validation point across our cohort which lends greater assurance that the prognostic factors we have identified are significant and clinically relevant.

The overall survival durations in our study are consistent with published literature. Our data shows patients with multiple metastases and WBRT have a median overall survival of 1.5 months and those with 1-3 metastases and WBRT have a survival of 3.6 months. In the UK QUARTZ study of 151 patients, a median overall survival between 49 to 51 days was observed, where $68 \%$ of patients had 1-3 metastases (Langley et al., 2013).

The median overall survival of 3.4 months seen in our study is shorter than the reported mean overall survival of 9.0 months in the subset of patients with lung primary in Iran (Akhavan, 2014). Differences are likely due to the definition of overall survival. This study defined overall survival from the the date of treatment initiation for BM to death from any cause, while in the Iranian study, overall survival was defined as time from date of diagnosis of BM to death from any cause. Furthermore, this study reported median whereas the latter study reported mean overall survival.

This study confirms the survival benefit with aggressive local therapy in appropriately selected patients. Furthermore the significant independent prognostic factors found in this study are consistent with the results of a large, multi-institutional retrospective analysis done in the United States (Sperduto et al., 2010).

Limitations of this study include its retrospective design, lack of assessment for intracranial control, lack of data on biomarker information, data on use of molecular targeted therapy (MTT), quality of life, acute and late radiation toxicity. We recommend prospective study with inclusion of biomarker, data on use of MTT and measurement of quality of life to further evaluate prognostic factors in NSCLC patients with brain metastases.

In summary, ECOG score $\leq 2$, presence of seizure, oligometastatic disease treated with aggressive local therapy (surgery/SRT) and the use of post-therapy systemic treatment are significant independent prognostic factors for overall survival in our group of patients with NSCLC and brain metastases. We recommend further evaluation of the role of aggressive local therapy either surgical resection or SRS/SRT in patients with BM and NSCLC.

\section{Acknowledgements}

We would like to express our sincere gratitude to all the patients, nurses, radiation therapists and support staff involved. We are thankful to Mr Chung Rui-En for his help in data management.

\section{References}

Akhavan A, Binesh F, Heidari S (2014). Survival of brain metastatic patients in Yazd, Iran. Asian Pac J Cancer Prev, $\mathbf{1 5}, 3571$.

Cai Y, Wang JY, Liu H (2013). Clinical observation of whole brain radiotherapy concomitant with targeted therapy for brain metastasis in non-small cell lung cancer patients with chemotherapy failure. Asian Pac J Cancer Prev, 14, 5699-703.

Ceresoli GL, Reni M, Chiesa G, et al (2002). Brain metastases in locally advanced nonsmall cell lung carcinoma after multimodality treatment: risk factors analysis. Cancer, $\mathbf{9 5}$, 605-12.

Chang EL, Wefel JS, Hess KR, et al (2009). Neurocognition in patients with brain metastases treated with radiosurgery or radiosurgery plus whole-brain irradiation: a randomised controlled trial. Lancet Oncol, 10, 1037-44.

Duan L, Zeng R, Yang KH, et al (2014). Whole brain radiotherapy combined with stereotactic radiotherapy versus stereotactic radiotherapy alone for brain metastases: a meta-analysis. Asian Pac J Cancer Prev, 15, 911-5.

Gaspar L, Scott C, Rotman M, et al (1997). Recursive partitioning analysis (RPA) of prognostic factors in three Radiation Therapy Oncology Group (RTOG) brain metastases trials. Int J Radiat Oncol Biol Phys, 37, 745-51.

Gehan EA, Walker MD (1977). Prognostic factors for patients with brain tumors. Natl Cancer Inst Monograph, 46, 189-95.

Hart MG, Grant R, Walker M, et al (2005). Surgical resection and whole brain radiation therapy versus whole brain radiation therapy alone for single brain metastases. Cochrane Database Syst Rev, 1.

International Agency for Research on Cancer (2014). GLOBOCAN 2012: estimated cancer incidence, mortality and prevalence worldwide in 2012. World Health Organization. http://globocan. iarc. fr/Pages/fact_sheets_ cancer. aspx. Accessed on 20 September 2014.

Langley RE, Stephens RJ, Nankivell M, et al (2013). Interim data from the Medical Research Council QUARTZ trial: does whole brain radiotherapy affect the survival and quality of life of patients with brain metastases from non-small cell lung cancer? Clinical Oncol, 25, 23-30.

Law A, Karp DD, Dipetrillo T, Daly BT (2001). Emergence of increased cerebral metastasis after high-dose preoperative radiotherapy with chemotherapy in patients with locally advanced nonsmall cell lung carcinoma. Cancer, 92, 160-4.

Liu WJ, Zeng XT, Qin HF, et al (2012). Whole brain radiotherapy plus chemotherapy in the treatment of brain metastases from lung cancer: a meta-analysis of 19 randomized controlled trails. Asian Pac J Cancer Prev, 13, 3253-8.

Natukula K, Jamil K, Pingali UR, et al (2013). Survival analysis in advanced non small cell lung cancer treated with platinum based chemotherapy in combination with Paclitaxel, gemcitabine and Etoposide. Asian Pac J Cancer Prev, 14, 4661-6.

Oken MM, Creech RH, Tormey DC, et al (1982). Toxicity and response criteria of the Eastern Cooperative Oncology Group. Am J Clinical Oncol, 5, 649-56.

Patchell RA, Tibbs PA, Walsh JW, et al (1990). A randomized trial of surgery in the treatment of single metastases to the brain. N Engl J Med, 322, 494-500.

Patil CG, Pricola K, Sarmiento JM, et al (2012). Whole brain radiation therapy (WBRT) alone versus WBRT and radiosurgery for the treatment of brain metastases. Cochrane Database Syst Rev, 9.

Postmus PE, Smit EF (1999). Chemotherapy for brain metastases of lung cancer: a review. Ann Oncol, 10, 753-9. 
Weng Heng Tang et al

Priestman TJ, Dunn J, Brada M, Rampling R, Baker PG (1996). Final results of the Royal College of Radiologists' trial comparing two different radiotherapy schedules in the treatment of cerebral metastases. Clin Oncol, 8, 308-15.

Sperduto PW, Berkey B, Gaspar LE, Mehta M, Curran W (2008). A new prognostic index and comparison to three other indices for patients with brain metastases: an analysis of 1,960 patients in the RTOG database. Int J Radiation Oncol Biol Phys, 70, 510-4.

Sperduto PW, Chao ST, Sneed PK, et al (2010). Diagnosisspecific prognostic factors, indexes, and treatment outcomes for patients with newly diagnosed brain metastases: a multiinstitutional analysis of 4,259 patients. Int J Radiat Oncol Biol Phys, 77, 655-61.

Van Vulpen M, Kal HB, Taphoorn MJ, El Sharouni SY (2002). Changes in blood-brain barrier permeability induced by radiotherapy: implications for timing of chemotherapy? Oncol Reports, 9, 683-8.

Zeng YD, Zhang L, Liao H, et al (2012). Gefitinib alone or with concomitant whole brain radiotherapy for patients with brain metastasis from non-small-cell lung cancer: a retrospective study. Asian Pac J Cancer Prev, 13, 909-14. 